\title{
Public Perceptions of Cultured Meat Pseudonyms
}

\author{
Jackson Webster ${ }^{1}$ and Patricia Talarczyk ${ }^{1 \#}$ \\ ${ }^{1}$ Mentor High School, Mentor, OH, USA \\ \#Advisor
}

ABSTRACT

The titles used for cultured meat vary drastically and a single name needs to be found. This would allow for a clear name to be established in order to appeal to consumers and appealing in this way would help to best cultivate the benefits of this meat that could help the environment and economy. To measure this, a 7-point likert scale in a survey with many of these titles and various company logos was employed. Several analysis tests (ANOVA repeated measures and difference of means) were thereafter used to analyze the data found. It was then concluded that crueltyfree meat was, by a large margin, statistically significantly above the other titles and should be used for further practice and sales.

\section{Public Perceptions of Cultured Meat Pseudonyms}

\author{
What Is Cultured Meat?
}

Cultured meat, lab-grown meat, in-vitro meat, and cruelty free meat are all names that the average consumer likely is not very cognizant of. When one first sees them, they would often envision that these are all separate products; however, all of these names are for the exact same product. Indeed, cultured meat is a nascent product, and with its new nature comes a slew of marketing terms and ideas that have all become contradictory of one another all in proposition of the same type of product, but this leads to the question, what is cultured meat? Cultured meat is a type of meat formed from the growth of meat out of stem cells taken from cows, pigs, or other livestock to create meat that requires almost no harm to these animals (Hawaleshka 2005). This meat is grown completely in a lab environment in dishes that use sterilized equipment to make sure the process is as clean as it can possibly be. This has already been seen in the preexisting creation of some cultured meats, such as the original burger created by Mark Post and his associates (Kupferschmidt 2013). This burger was the first to prove that this method would be viable, despite its high costs, and also demonstrated the viability of this technique in removing the many diseases that can be present in meats due to how it can be controlled as it grows.

The benefits present in the use of this technique have caused many to show interest due to its strength as a well-controlled solution to animal cruelty and environmental problems that can be seen in conventional farming. Due to this reduced use of animals throughout the process, this meat has also widely been accepted far more than normally farmed meat by vegetarians and vegans (Chauvet 2018). The lowered amount of cruelty present in the farming of this meat compared to how animals are normally raised in packed conditions to be sent off to slaughter later in their lives appeals to the vegans and vegetarians as this only requires a small amount of interaction with the animals through the collection of the cells, and afterwards, the animals can live out far better lives without having to be packed with many other animals. There are also many environmental benefits present with the use of cultured meat as it, in combination with green energy sources, would drastically cut the amount of land and greenhouse gas emissions that farming is responsible for as farming is currently one of the largest polluters (Mattick, C. S. 2018). This has led many who are in favor of reducing climate change to bandwagon behind increased research of this meat, and to support its future expansion and research, but this meat is still mostly in development and has very little market experience so far. 


\section{The Expansion of Cultured Meat Into The Market}

Cultured meat has very little market presence thus far, but many companies have begun to form in order to begin the production and selling of this meat. The chief three companies among these are Mosa Meats (which is partially run by Mark Post, who created the first lab-grown burger), Finless Foods, and Memphis meats. These three startups have taken their combined efforts into conducting more research about cultured meat and its possible greater use abroad in the market. Memphis Meats is currently getting closer to this goal as they have begun to hone in on meatballs as a product that works with this setup quite well and have researched this specifically extensively (Burningham 2016).

This enthusiasm has been in part halted, however, due to the extensive failure of genetically modified organisms (GMOs) in the past that the cultured meat companies are trying desperately to avoid. In the past, GMOs experienced much trouble with their marketing and helping people to understand their products. Many people did not understand what GMOs meant and how they did not harm people to consume nor their creation from a safe genetic process, which led to much drama between producers and consumers for these products as well as legal trouble for those who sold GMO products. This thereafter led to battles in legislation about how these products should be able to be marketed and labeled, which ultimately drove off more customers from buying these products (Lee 2016). This legal conflict painted the products in a bad light and made them appear untrustworthy to the general public and caused their marketing to fail. Another reason their marketing failed was due to their mismanagement of how their products were labeled between different companies that made these products (Kling 2014). This situation, which is now being reflected in cultured meat's many names, led to their ultimate demise as people did not understand what their products meant and were instead scared off by these terms. The current mirroring of this situation in cultured meat could spell disaster and shows why market research is needed on the topic.

\section{Current Market Research On Cultured Meat}

Some research for the marketing of cultured meat is present on how the information presented to consumers can change how they feel about the consumption of cultured meat. The average consumer tends to be mostly unaware of cultured meat, how it is created, and its benefits, which leads to complications when measuring consumers' attitudes. To determine how much this effect will affect how consumers view cultured meat, scientists have used studies where a survey about cultured meat will be given, followed by information, and then followed by the same survey again. In these studies, it has been proven that increasing the knowledge of consumers about cultured meat will have a positive impact on how they feel about this meat (Dupont \& Fiebelkorn 2020). In fact, the increased information about these meats has even proven to make them seem more appetizing in a tasting context (Rolland et al. 2020). This phenomenon shows how consumers may be scared off by the term cultured meat due to how it sounds scientific and unnatural, further mirroring how GMOs were viewed, which demonstrates how these scientific terms may not be the best for the marketing of these products.

Additionally, outside of environmentalists and those who wish for better treatment of animals, not much research has been conducted as to how many groups feel about cultured meat due to its relatively recent origins. This means that while we understand that there is somewhat of a gap between consumers and scientists for the scientific terminology used (Tomiyama et al. 2020), we are unsure of what other problems may persist in its marketing. This does not bode well for the future of cultured meat marketing, and the situation GMOs faced in the past shows that this may be an especially prevalent issue due to the lack of understanding the public may have. This means that in order for cultured meat to become a viable product for the market, more research will have to be done into the sales of it. 


\section{Informational Gap Addressed in This Paper}

The nomenclature of cultured meat is an especially prevalent issue in this marketing that has yet to be analyzed by experimental data. Generally, it is inferred from the failure of GMOs that terms such as "cruelty-free meat" will be the best for marketing to consumers as it has a very environmentally friendly connotation and denotation, but no research has been fully conducted to measure this. Currently, the wide variety of terms used to market these products boasts a very deleterious effect on the future of these companies if a unified term is not found.

This harmful effect would also spread to cover the world as the disregard that could be generated for this meat would spell disaster for climate change. Our meat consumption has been steadily rising, doubling in the last thirty years and being set to double again in the next thirty, so a solution to this increased consumption is clearly needed (New Scientist 2020). The current farming methods would cause massive increases in greenhouse gas emissions and space taken for farming if they were to continue to be used, which would harm the world a tremendous amount. Cultured meat, however, takes up far less space and emits far fewer greenhouse gasses, meaning it would be able to effectively help provide this meat for the future and help save the environment (Zanteson 2020).

Given the current condition of cultured meat, I aim to, using a survey system designed to measure both demographic information and consumer opinion, determine which terms are best for the marketing of cultured meat in this study. This would then allow for continued study to evolve on this topic before settling on the terms that companies can use to avoid consumer confusion as well as spreading this meat, and its beneficial effects, to as many people as possible.

\section{Methods}

\section{The Survey and Its Structure}

This survey and the created questions were created to measure three main things, demographics, knowledge of cultured meat and opinions of the subjects on the several labels for lab grown meat provided. Before this, however, the survey began with a form asking for the informed assent of the participants after giving them a description of the study and the risks in order to make sure participants understood the study and what they were completing. This then brought participants to the demographics questions which were poised to find information about the person taking the test by having them assess several parts of their backgrounds. These included age, economic standing, and political standing, and these questions were analyzed and found from a common demographics source to ensure that they will be best to help assess these details for my survey (Wroblewski, 2019).

Next, the survey asks subjects to complete a small questionnaire about the use of lab-grown and cultured meat in order to assess their knowledge of the two and to see whether or not they know they were the same. These questions were tailored by myself to ask the same questions about these two names and to ask general questions about their use and benefits. These questions were written in such a way that they would accurately assess this using methods described in research conducted before this study. It was found that, "knowing the right questions and writing them clearly and specifically are important things to consider in writing survey questions" (Shank, 2008). With this insight, as well as more gained from further investigation on question creation, the questions were able to be formed and tailored to measure knowledge for both meats using the exact same format to avoid leading those unaware to the conclusion that these meats are the same or different. For instance, one pair of questions posed whether cultured/labgrown meat was more beneficial to the environment than conventional farming. This question was created due to how knowledge of the benefits of this meat environmentally has shaped opinions about it drastically (Post 2020). This question also provides these two different titles for the meat in separate questions, and this can indicate that people may be unaware that the meats are synonymous if the same answer is chosen. The knowledge of a participant on these meats is measured in this survey because of how it can, as demonstrated by the question pair previously discussed, 
change one's opinions on cultured meat and, thus, the different names and logos (Bekker et al., 2017). This means that questions needed to be created to determine if the subjects were knowledgeable to account for this change when ratings came into play.

Lastly, the survey presented several different names and brands for the sale of this meat and asked participants to rate them on a seven point Likert scale. Several different names and brands were chosen due to their possible different viewing by subjects. Names that suggest less scientific practices (cruelty free meat) may elicit a different response than those that do (lab-grown meat), and this is a large portion of what required observation. The different logos also have different elements in their designs such as more natural themes or more scientific themes that also required analysis, which can be seen in Figure 1 and Figure 2.

\section{:a: mosameat}

Figure 1. Logo Of Mosa Meat

Note. This logo shows more scientific themes throughout its design. Note the orange and blue colour scheme of the logo as well as the spots (almost resembling those of a petri dish) alongside the text. Sourced from Mosa Meat (2016).

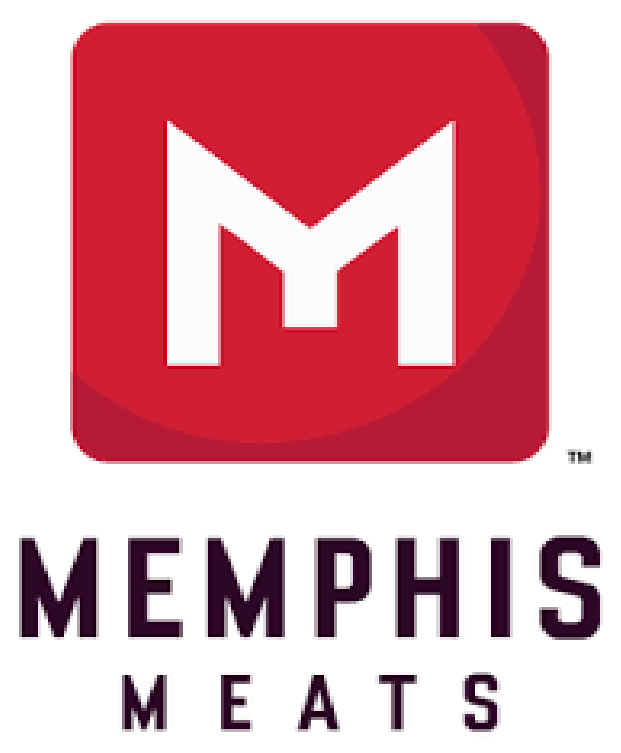

Figure 2. Logo Of Memphis Meats

Note. There appears to be little involved with this logo. The logo is quite simple and only portrays a large letter M surrounded in red with the company title underneath.

All of these different descriptors and elements can be found in the appendix of my survey. These would then contribute to different opinions on these items, and could cause bias in selection from the way these titles are presented. The ratings of these different things are believed to be different due to the way that they are phrased, and these were presented all in the same way (simply stated on the quiz with no lead up) to avoid bias. Comparison of these different ratings and names can then be justified to compare how people feel about these titles. From this analysis, the 
differences in demographics, knowledge, and opinions can then be evaluated and tested for any correlation given these results, determining if current marketing strategies are being used effectively or not in order to avoid previous failures.

\section{Data Collection and Planned Demographics}

The survey was distributed to subjects through the use of a digitized form on Google Forms and an email in order to ensure safety, reach, and confidentiality with the survey. Firstly, a digital format being passed through an email ensured the safety of those taking the survey through how it did not require any person-to-person interaction between myself and those taking the survey to allow them to fill it out. This then helped to limit the possible spread of disease by separating people and not sharing physical materials between them and myself. Due to the current pandemic, the survey could not be placed in public spaces, such as grocery stores, and thus these adjustments were necessary to keep the study as safe as possible, and they have allowed for almost no spread to be possible from my study. This use of an email and Google Form also increased the possible reach by which the survey was able to be distributed out to subjects. The email would be able to be distributed to many people, which would allow for a diverse group to be selected for the survey through the distribution of the survey outwards. This could also allow for more demographics to be reached from how the survey could be distributed out of the local area. Lastly, the design of the survey on this Google Form also helped to ensure that the method by which the data was collected was in a secure and confidential way. The survey asked several demographic questions, but it did not require any to be answered, allowing for participants to only answer what they wished throughout the survey and not pressuring them into answering. The survey also did not collect any names or emails of individuals who the email was distributed to, and this helps to ensure that the data was confidential due to how the responses could not be tracked or connected to the one who filled out by either of these factors. Keeping the confidentiality of the subjects then held this up to the standards set to keep integrity in the review of my responses as it does not allow for pinpointed responses to be found or any action against one who takes the survey from this.

This survey format was intended to reach an audience of suburban, middle-aged people who would be the most likely to purchase these products. Those who frequent supermarkets or other stores would be important to analyze in this survey as this is where the meat would be sold, and thus, those who frequent supermarkets the most would be the most important to reach for the marketing of these products. This has led into an investigation wherein it was determined that the prime subjects for this survey would be middle-aged (37-52 years old) individuals who, according to a study published on Acosta, are likely to spend more on groceries than any other generation (2018). Additionally, this survey aims to measure how certain demographics may also view these products differently. It is for this reason that the survey does not aim for specific quality of life measures, incomes, and ethnicities among others as analyzing how these differ may be very important for the results of the survey; henceforth, this survey aims to survey as many of these groups as possible to determine any correlations that could impact this marketing as well. Different groups with different backgrounds may have different education and cultures that lead to these different opinions, and they may know differing amounts about this meat, which has already been determined to influence the data, and these factors are also important to consider. This would then lead to a healthy mix for my final results to be analyzed for differences.

\section{Results}

\section{Key Limitation}

Before any statistical analysis, an overview of how the method was able to be carried out should be introduced. Despite efforts made to spread the survey to many individuals, it should be noted that the survey was only able to obtain 30 responses. In an attempt to provide the survey to a variety of individuals, a variety of schools in northeast Ohio were 
contacted, emailing staff to obtain responses; however, this proved quite unsuccessful. Given that I was able to obtain 30 responses, my work is far less likely to be sufficiently representative of an average consumer population, but the sample size is still large enough to run a statistical analysis upon. This means that my sample would contain a normal distribution of responses over the people I was able to communicate to but the sample may not perfectly fit a larger population, such as the entirety of the U.S. population.

\section{Knowledge And Demographics}

Knowledge and demographics would have a different approach taken for their measurements as correlation needed to be measured for the relation of the two variables. Knowledge was first measured and a scatter plot (Figure 3) was created in order to determine if the knowledge of the participants (as was measured through the knowledge section of the survey) increased the value at which the participants rated the various meat products through titles, company titles, and logos alike. The mean score of the rating of all of the different facets of this product would then be taken and combined to form a complete average. This was then connected to the score of the participant on the knowledge section and a correlation coefficient was found. This value was found to be quite low, at 0.55 , but a definite positive trend (shown via the trendline) is to be noticed as the ratings do progressively become more accepting of the products with a higher knowledge score.

\section{Questions Correctly Answered On Knowledge Scale vs. Average Rating}

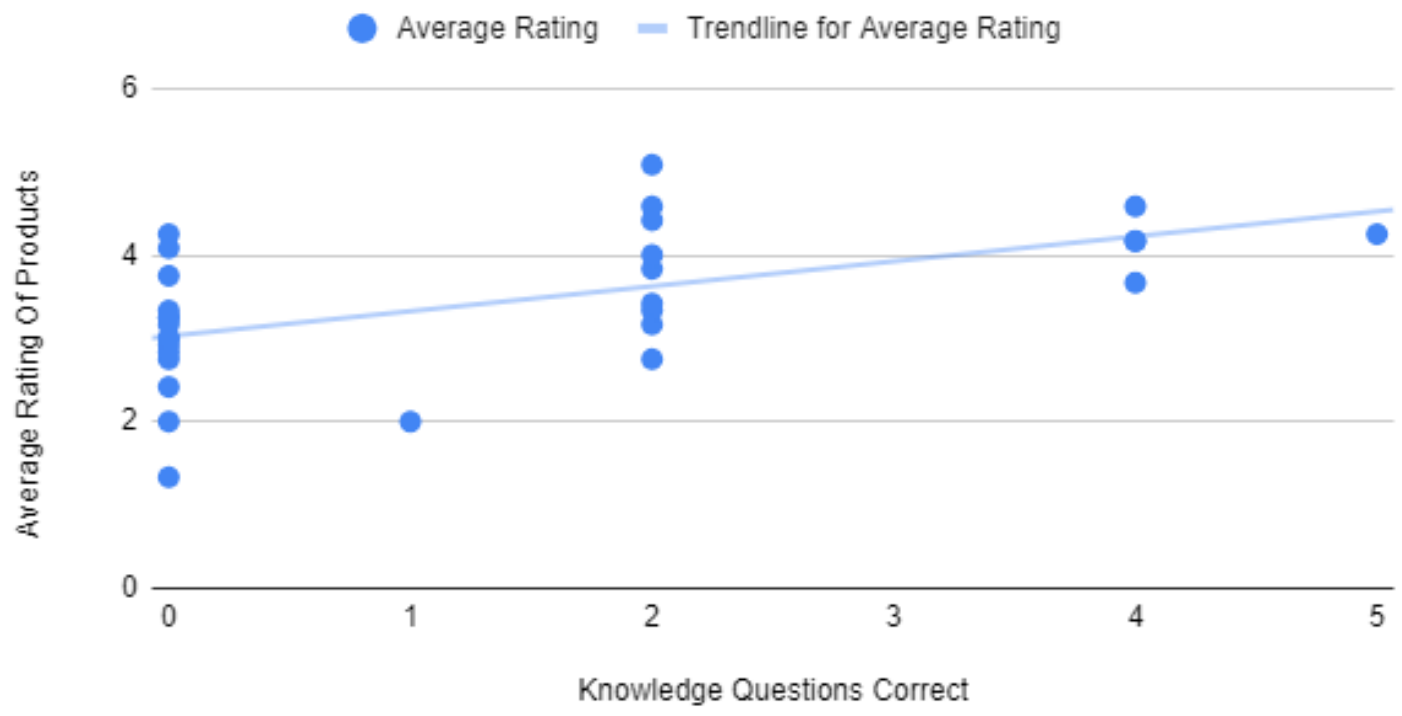

Figure 3. Scatterplot Showing The Distribution Knowledge Rating V. Total Average Rating

Note. There appears to be a positive correlation between these two values that is confounded slightly by those with little knowledge having varying opinions. Overall, a positive trend can be seen through most of the points on the graph.

As for measuring whether subjects were knowledgeable of the lab-grown and cultured meat being different, only eight of the thirty participants kept answers consistent for both meats without selecting they did not know for more than $50 \%$ of the reponses. This is noted independently of questions being answered correctly as one may know the meats are the same but be unaware of what they do. Accounting for only correct answers would remove this case and skew the data. 
The demographic-based data that was measured was not found to have much correlation with the ratings in the sample. Tests for the difference of the means of these different groups were run in order to determine if any correlation may be present between various factors of a person's background that may influence them. Given birth sex, income, race, and more, the demographics did not have a high correlation coefficient in any given background, showing that these differences likely do not change one's view of these products at all and does not contribute to a more positive or negative view of these ideas. Additionally, it should be noted that this lack of correlation may be a result of the low sample size of this paper as few individuals with each background were able to be analyzed in most cases due to the differences among the participants only being represented by 30 individuals who could only embody certain demographics with a few of their members.

\section{Titles}

Upon analysis of the titles, it was first most important to note if there was any significance among the different titles presented in order to show if these different titles caused change in the rating given by various individuals. To this extent, a test for significance was performed in order to determine if the hypothesis that a different title being presented would stir up different emotions would need to be evaluated. A repeated measures ANOVA test was conducted on the data in order to determine if this effect caused measurable variance among the samples and it was found to have significant difference among the samples in changing the opinions of consumers at a level less than 0.01 (Statistics Calculators, n.d.).

After this point, tests for the analysis of means would need to be run in order to determine the superlative title in this area as this indicates that this title would need to be applied in further use for marketing to the general public and best supporting the sales given. From this, there were two titles that were clearly above the others, cultured meat and cruelty-free meat as is shown in Figure 4 below.

\section{Average Ratings Of Various Titles On A 7-Point Likert Scale}

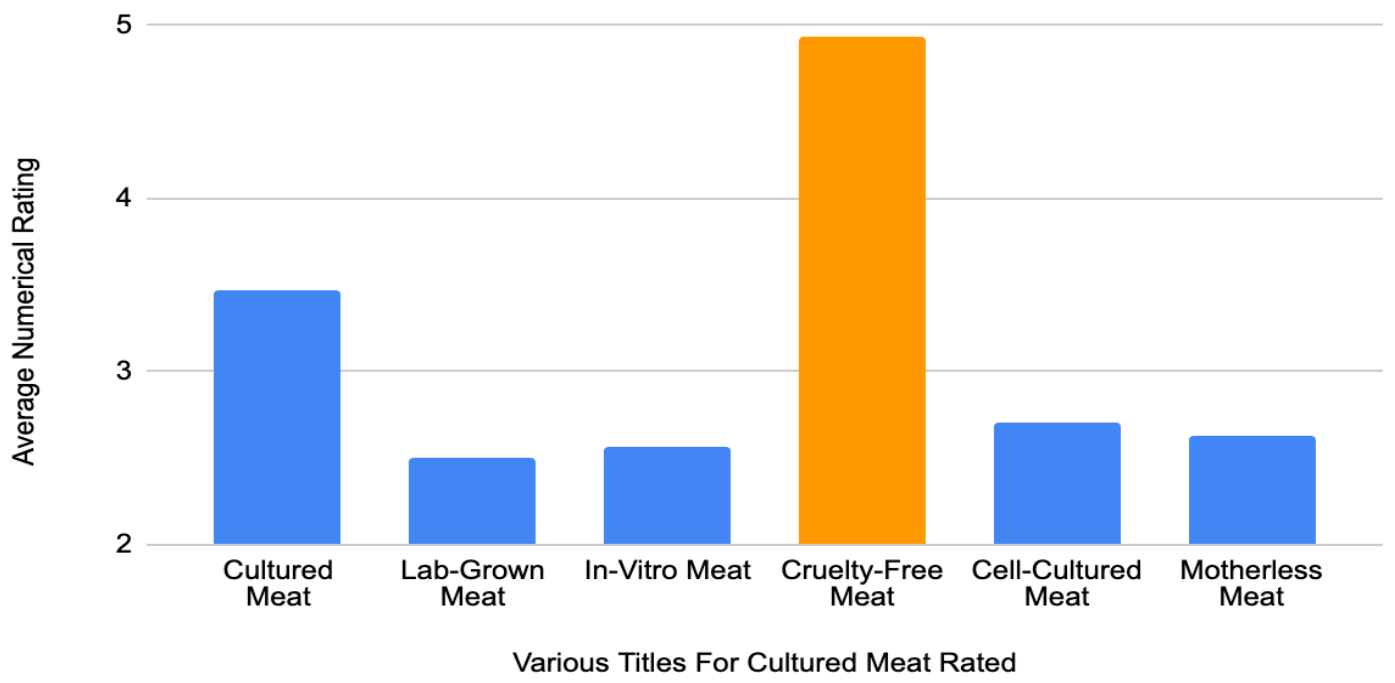

Figure 4

Bar Graph Showing The Distribution Of The Mean Ratings Of The Cultured Meat Titles

Note. While most others averaged around 2.5, cultured meat and cruelty-free meat were much higher at 3.467 and 4.933 respectively. 
These two different titles would then be compared to the other titles given and one another. A t-test analyzing the difference between the means of the meats, comparing one to another one at a time, would be conducted in order to show if any significant differences were present. From this, cruelty-free meat would be established as the clear best title. It was proven to be statistically significant in having a higher rating than every single other title for this meat (all were conducted at $\boldsymbol{\alpha}<0.01$ ). Cultured meat was also proven to be a valuable name as it scored higher than all of the other meat titles that were not cruelty free meat with the same statistical test being run. This meat was also proven higher than all but cruelty-free meat at a $\boldsymbol{\alpha}<0.01$ as well. The remaining titles were compared to one another and no significance in the differences of their rating were found. Additionally, the variances of these meats as seen in Table 1 below, which displays the various means, variances, medians, and more of each title, is quite low, which shows that these ratings are seemingly quite consistent.

Table 1. Summary Statistics For Cultured Meat Titles

\begin{tabular}{llllllll} 
Statistics & \multicolumn{6}{l}{ Different Titles For This Meat } \\
\cline { 2 - 8 } & $\begin{array}{l}\text { Cultured } \\
\text { Meat }\end{array}$ & $\begin{array}{l}\text { Lab- } \\
\text { Grown } \\
\text { Meat }\end{array}$ & $\begin{array}{l}\text { In-Vitro } \\
\text { Meat }\end{array}$ & $\begin{array}{l}\text { Cruelty- } \\
\text { Free Meat }\end{array}$ & $\begin{array}{l}\text { Cell- } \\
\text { Cultured } \\
\text { Meat }\end{array}$ & $\begin{array}{l}\text { Motherless } \\
\text { Meat }\end{array}$ & Total \\
\hline Mean & 3.46666666 & 2.5 & 2.5666666 & 4.93333333 & 2.7 & 2.63333333 & 3.13333333 \\
Median & 3.5 & 2 & 2.5 & 5 & 3 & 3 & 3 \\
Mode & 3 & 1 & 1 & 7 & 1 & 1 & 1 \\
Range & 6 & 4 & 5 & 6 & 4 & 4 & 6 \\
Standard & & & & & & 1.7 & \\
Deviation & 1.56983050 & 1.35824430 & 1.5905612 & 1.76035524 & 1.3169976 & 1.29942516 & 1.70899689 \\
$\mathrm{~N}$ & 30 & 30 & 30 & 30 & 30 & 30 & 180 \\
\hline
\end{tabular}

\section{Companies}

The company titles and logos would also be ushered through a similar process for this statistical analysis. The company titles, however, would stand to prove far less fruitful in their significance as the data found for the difference in the ratings among these different company titles was found to be insignificant. Additionally, when viewing the data, one can note that there does not seem to be much difference in the average ratings of all of these companies, and this is demonstrated in Figure 5 below. 


\section{Average Ratings Of Company Titles And Logos On A 7-Point Likert Scale}

5

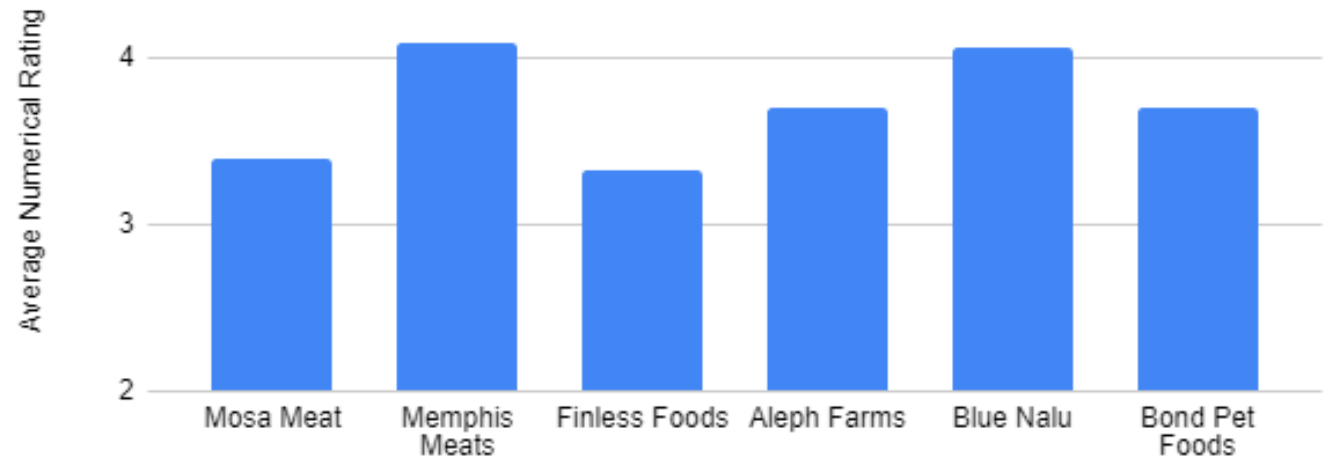

Various Titles And Logos Rated

Figure 5. Bar Graph Showing The Distribution Of The Mean Ratings Of The Cultured Meat Companies And Logos

Note. Though the averages do vary across these titles, and some may seem better than others, the range of the means is only 0.77 , which is quite small and indicates that the means do not vary much.

The many company titles and logos did show that some were better received as data points with higher values can be seen, but the majority of the data is shown to not have much substance due to the insignificance among the points. Additionally, the companies did not have any significant data when compared to one another. Similarly to the analysis for the titles, the logos and company titles would also go through a variety of $t$ tests for significance among the different ideas expressed; however, none would be proven to be significant above the others at a level lower than $\boldsymbol{\alpha}<0.1$. From this, and the relatively low variances (see Table 2), it can be deduced that the company titles and logos also are quite consistent throughout the sample.

Table 2. Summary Statistics For Cultured Meat Company Titles And Logos

\begin{tabular}{llllllll} 
Statistics & \multicolumn{7}{l}{ Company Titles } \\
\cline { 2 - 7 } & Mosa Meat & $\begin{array}{l}\text { Memphis } \\
\text { Meats }\end{array}$ & $\begin{array}{l}\text { Finless } \\
\text { Foods }\end{array}$ & $\begin{array}{l}\text { Aleph } \\
\text { Farms }\end{array}$ & Blue Nalu & $\begin{array}{l}\text { Bond } \\
\text { Foods }\end{array}$ & Pet Total \\
\hline Mean & 3.4 & 4.1 & 3.3333333 & 3.7 & 4.0666666 & 3.7 & 3.7166666 \\
Median & 4 & 4 & 3.5 & 4 & 4 & 4 & 4 \\
Mode & 4 & 4 & 4 & 4 & 5 & 4 & 4 \\
Range & 5 & 6 & 5 & 5 & 5 & 5 & 6 \\
Standard & & & & & & & \\
Deviation & 1.2205143 & 1.1551981 & 1.184187 & 1.1788363 & 1.0806553 & 1.1188047 & 1.1783664 \\
$\mathrm{~N}$ & 30 & 30 & 30 & 30 & 30 & 30 & 180 \\
\hline
\end{tabular}




\title{
Discussion
}

\author{
Implications Pertaining to Knowledge Measures
}

The correlation between the measured knowledge and the opinions of the participants being low is a surprising result of my study. Given that the correlation coefficient was smaller than it would be thought to be, this data may point towards knowledge being less important than previously thought to influencing the views of the public on these products. Previous research into this field has indicated that the knowledge of participants can be very important in shaping what they think of these products as they may be more likely to support them if they are aware of more about the meat and its benefits; however, my survey (seen in the appendix) asks various simple questions about this meat and whether or not it is more beneficial than contemporary farming practices employed around the world, and it seems that more knowledge about this is not correlated very well with higher scores. This data provokes a variety of questions namely whether these questions were comprehensive enough to provide an analysis of the knowledge of the people, whether my sample of these teachers from northeast Ohio was different from most other samples analyzed to be differently predisposed to this meat, and if more confounding variables may be present that only a greater number of studies focused more on this topic can answer. Despite this low correlation coefficient, it can still be noted that there is a general trend of ratings increasing with knowledge that must be understood to still be indicative of former findings. This shows that the knowledge does likely influence the ratings of the people to some extent but it may be limited in some levels as many people may not know much about the meat and think it sounds favorable or understand the benefits but wave them away for other reasons. Increased research is still necessary to affirm this thought, however. As for the knowledge of the public on whether or not lab-grown and cultured meat are the same product, results seem to indicate that most are unaware of the products being the same. Only eight of the thirty participants kept answers consistent for both meats without selecting they did not know for more than $50 \%$ of the reponses. Thus low amount of responses favouring knowledge of the two being synonymous spells out quite clearly that many are unaware of these products being the same. This then shows further how the education of the public may be valuable as it can help to counteract this misinformation or lack of information as well. Further evidence pointing to more work to educate the consumers in this regard further supports the idea that marketing specialists should focus on this in their campaigns in order to best help people understand this product.

\section{Implications In Product Marketing}

This study clearly points to one title as the best for consumer acceptance, and that title is cruelty-free meat. This title was shown to be statistically significant in being higher than all of the other titles for the meat and accrued the highest ratings compared to all of the other titles as well as the company titles and logos. This means that this is very likely the title that should be employed going forward if this product is to best be accepted and used by common consumers, but the small sample size of this study may cause this to be incorrect. Thus, more inspection of this claim is necessary in order to fully state that it is the best title. A greater amount of research on this topic with a larger sample size would help to affirm this claim and provide the best avenue for selling this meat to the public.

As for the title and logo of the company, this section is seen to not be incredibly important in the marketing of this meat. The scores did not vary significantly between the various company titles and logos, but one trend that may be noticed is that the higher rated logos tended to more nondescript (Memphis Meat's large M) or more natural (Aelph Farms's name and logo) while technological or scientific logos were rated lower (Mosa Meat's and Finless Foods's logos which can be seen in the appendix along with all of the other logos previously mentioned). This suggests that consumers may be turned away by scientific theming on logos, which is something to consider in order to maximize customers as a worse logo could cause business to be lost. More testing in this sector is also necessary in order to confirm this finding given the small sample size. It also may be wise to attempt to test different logos that are not from 
real companies in order to determine if these elements can bring change more effectively as the logos could be based on established standards.

\section{Overall Implications for The Next Steps Of Meat Marketing}

This study provides sufficient data to claim that scientists should likely unify until the title cruelty-free meat. Despite small sample sizes, the mean for this value was so much larger than the others that it is quite indicative that this is likely the best title, in addition to its significance for all of the others. In a similar vein, the title and logo of a company was found to be largely insignificant in the view of a product, but a trend seems to indicate that moving away from technological names is likely a good idea, so this should be taken into account and employed as well. Lastly, my data indicates that the knowledge of subjects on the meat has a general, positive trend which indicates that an initiative to educate consumers through adverts or other means should likely be taken as increasing the knowledge of this meat could help to sell it. All of this data would then allow humanity to better reap the previously mentioned benefits of this meat and to use it well for the environment and ourselves. Cruelty-free meat is a vital part of our future due to the improvements it can provide, and this data helps to indicate a clearer way to reach these benefits that are necessary to help halt global warming and meet the demands of the future.

\section{References}

A lab-grown future. (2020). New Scientist, 245(3270), 3. https://doi.org/10.1016/s0262-4079(20)303742

Acosta. (2018). Acosta News And Publications. https:/www.acosta.com/news/acosta-s-the-why-behindthe-buytm-profiles-five-generations-of-grocery-shoppers

Bekker, G. A., Fischer, A. R. H., Tobi, H., \& van Trijp, H. C. M. (2017). Explicit and implicit attitude toward an emerging food technology: The case of cultured meat. Appetite, 108, 245-254. https://doi.org/10.1016/j.appet.2016.10.002

BURNINGHAM, G. (2016). The Plot to Beat Meat. Newsweek Global, 166(10), 46-49.

Chauvet, D. J. (2018). Should cultured meat be refused in the name of animal dignity? Ethical Theory \& Moral Practice, 21(2), 387-411. https://doi.org/10.1007/s10677-018-9888-4

Dupont, J., \& Fiebelkorn, F. (2020). Attitudes and acceptance of young people toward the consumption of insects and cultured meat in Germany. Food Quality \& Preference, 85, N.PAG.https:/ /doi.org/10.1016/j.foodqual.2020.103983

Hawaleshka, D. (2005). Coming right up: One test-tube T-bone. Maclean's, 118(47), 48.

Indie Bio (2017). Memphis Meat. https://indiebio.co/companies/memphis-meats/

Kling, J. (2014). Labeling for better or worse. Nature Biotechnology, 32(12), 1180-1183. https://doi.org/10.1038/nbt.3087

KUPFERSCHMIDT, K. (2013). Here It Comes ... The \$375,000 Lab-Grown Beef Burger. Science Now, 1. 
Lee, M. (2016). GMOs in the Internal Market: New Legislation on National Flexibility. Modern Law Review, 79(2), 317-340. https://doi.org/10.1111/1468-2230.12182

Mattick, C. S. (2018). Cellular agriculture: The coming revolution in food production. Bulletin of the Atomic Scientists, 74(1), 32-35. https://doi.org/10.1080/00963402.2017.1413059

Mosa Meat (2016). https://www.digitalfoodlab.com/en/foodtech-database/mosa-meat

Rolland, N. C. M., Markus, C. R., \& Post, M. J. (2020). The effect of information content on acceptance of cultured meat in a tasting context. PLoS ONE, 15(4), 1-17. https://doi.org/10.1371/journal.pone. 0231176

Statistics Calculators. Quick Statistics Calculators. (n.d.). https:/www.socscistatistics.com/tests/

Shank, P. (2008). Using Surveys to Improve Courses, Programs, and Instruction. Distance Education Report, 12(15), 4-5.

Tomiyama, A. J., Kawecki, N. S., Rosenfeld, D. L., Jay, J. A., Rajagopal, D., \& Rowat, A. C. (2020). Bridging the gap between the science of cultured meat and public perceptions. Trends in Food Science $\&$ Technology, 104, 144-152. https://doi.org/10.1016/j.tifs.2020.07.019

Wroblewski, M.T. (2019). What Are Examples Of Demographics. Chron. https://smallbusiness.chron.com /examples-demographics-65678.html

Zanteson, L. (2020). Cultured Meat: Designed for the Future: The future of meat is being developed in the laboratory with the promise of culinary satisfaction and sustainability. Environmental Nutrition, 43(4), 6. 